\title{
Féeries
}

Études sur le conte merveilleux, XVII ${ }^{\mathrm{e}} \mathrm{XIX} \mathrm{X}^{\mathrm{e}}$ siècle

\section{Les contes médiévaux et les contes de fées modernes}

Medieval Tales and Modern Fairy Tales

\section{Ruth B. Bottigheimer}

\section{(2) OpenEdition \\ 12 Journals}

Édition électronique

URL : http://journals.openedition.org/feeries/753

ISSN : 1957-7753

Éditeur

UGA Éditions/Université Grenoble Alpes

Édition imprimée

Date de publication : 31 octobre 2010

Pagination : 21-43

ISBN : 978-2-84310-182-3

ISSN : 1766-2842

\section{Référence électronique}

Ruth B. Bottigheimer, «Les contes médiévaux et les contes de fées modernes », Féeries [En ligne], 7|

2010, mis en ligne le 31 juillet 2011, consulté le 07 septembre 2020. URL : http://

journals.openedition.org/feeries/753

\section{(c) Féeries}




\section{Ruth B. Bottigheimer}

Research Professor Department of Comparative Literary and Cultural Studies, State University of New York, Stony Brook, USA

\section{LES CONTES MÉDIÉVAUX ET LES CONTES DE FÉES MODERNES*}

ELON LA SAGESSE CONVENTIONNELLE, on fait généralement remonter les origines du conte de fées au Moyen Âge. Les spécialistes du $\bigcup$ conte concluent donc généralement que tel ou tel conte est la forme médiévale d'un conte de fées moderne. De plus, les recueils de contes médiévaux internationaux, tels que Fairy Tales from Before Fairy Tales ${ }^{\mathrm{I}}$, Märchen des Mittelalters ${ }^{2}$ ou bien Formes médiévales du conte merveilleux ${ }^{3}$, portent des titres qui sous-entendent l'existence de contes modernes à l'époque médiévale.

Lassimilation de certains contes médiévaux à des contes de fées modernes tient en général à deux pré-conceptions. La première repose sur une définition inexacte des termes respectifs : conte de fées, Märchen et fairy tale. Je débats de cette question terminologique en détails dans "Fairy Godfather, Fairy-Tale History, and Fairy-Tale Scholarship" ${ }^{4}$, par conséquent ici je ne mentionnerai que brièvement la distinction que je fais

* Je souhaite exprimer mes vifs remerciements à Sophie Raynard-Leroy à qui j'ai demandé en urgence de traduire cet article depuis l'anglais et qui a suspendu toute autre activité pour s'y consacrer durant un intense échange de trois jours.

I. J. Ziolkowski, dans Fairy Tales from Before Fairy Tales (Ann Arbor Michigan, University of Michigan Press, 2006) discute de six contes qu'il qualifie de contes de fées. Quatre d'entre eux sont des "folktales» (c'est-à-dire, pas des contes de fées; voir plus loin pour les différences entre "folktales» et "fairy tales»), l'un d'eux est une légende religieuse, et l'autre, Asinarius, est un roman médiéval abrégé. Ce dernier ressemble quelque peu à un conte de fées moderne, mais il ne comporte pas ces composantes essentielles qui sont la souffrance du héros, son accomplissement de tâches et sa traversée d'obstacles.

2. A. Wesselski (éd.), I925.

3. J. Berlioz, C. Brémond et C. Velay-Vallantin, Formes médiévales du conte merveilleux, Paris, Stock, I989.

4. «Fairy Godfather, Fairy-Tale History, and Fairy-Tale Scholarship: A Response to Dan BenAmos, Jan M. Ziolkowski, and Francisco Vaz da Silva», Journal of American Folklore, 123.490 (Fall 20Io), p. 447-496, ici p. 46I-466. 
en anglais entre "folktales», "fairyland fictions", "restoration fairy tales» et "rise fairy tales». Les "folktales» (contes du peuple, plutôt que folkloriques au sens où les folkloristes l'entendent) sont des contes dont les protagonistes sont des gens ordinaires du peuple, qui font très souvent l'expérience d'une amélioration temporaire de leur statut, mais qui retournent à leur état d'origine à la fin du conte. Les «fairyland fictions» (fictions féeriques) présentent toujours quant à elles des mondes parallèles, l'un humain et l'autre féerique, entre lesquels évoluent les protagonistes, souvent avec des résultats désastreux pour les humains. Les "restoration fairy tales» (contes de restauration) décrivent la perte de statut d'un personnage royal, ses souffrances, l'accomplissement de tâches et la traversée d'épreuves insurmontables par ce personnage, et son retour final sur le trône grâce à un mariage magiquement orchestré avec un autre personnage royal. Enfin, les "rise fairy tales» (contes d'ascension) présentent un héros ou une héroïne pauvre, qui souffre, surmonte des épreuves, et qui parvient à accéder au statut royal et à la richesse grâce à un mariage magiquement orchestré avec un personnage royal. Le fait est qu'en anglais un fairy tale comme The Little Red Riding Hood s'appelle un conte de fées parce qu'il apparait dans le recueil communément appelé Grimm's Fairy Talees, alors qu’en français la présence d'un loup, qui parle dans le conte équivalent intitulé Le Petit Chaperon rouge, fait de ce conte un conte merveilleux. Or, en réalité, que ce conte apparaisse dans la version des Grimm ou de Perrault, il s'agit clairement d'un conte d'avertissement.

Le second problème est de même un problème de définition par nature : il a à voir avec ce qu'on entend par un conte et l'histoire qu'il raconte. On peut dire par exemple que les motifs principaux du conte Chat botté/Puss in Boots sont les suivants : un garçon, un chat botté, un mariage et une conclusion heureuse. Mais ce n'est pas le cas. Ainsi ces quatre éléments se trouvent dans la légende anglaise de Dick Whittington, qui diffère pourtant de celle-ci au niveau de l'histoire. Par exemple, les attributs félins du chat sont exagérés (dans la quantité de rongeurs qu'il peut consommer), mais ne sont pas magiques; d'une part, la fortune de Dick précède son mariage, d'autre part, il épouse une femme appartenant à la classe des commerçants londoniens. En revanche, le pauvre héros du Chat botté de Perrault possède un chat magique, plein de ressources, et qui parvient à lui faire épouser une princesse, le faisant ainsi accéder à une grande fortune. En d'autres termes, ce conte raconte l'histoire de l'ascension sociale et économique d'un héros pauvre par son accession au trône grâce à un mariage où la magie a eu sa large part. C'est aussi le cas dans les deux histoires dont dérive le Chat botté de Perrault, respectivement Gagliuso de Giambattista 
Basile et Costantino Fortunato de Giovan Francesco Straparola. De cette discussion introductrice, on peut facilement tirer que la signification culturelle et la désignation littéraire d'un conte - en tant que conte de fées, légende ou autre forme simples — dérivent du genre d'histoire qu'il raconte et non des motifs dont il est composé.

Je vais suggérer ici une proposition complètement différente : à la Renaissance — à savoir à l'époque où Straparola l'a écrite —, l'histoire (c'est-à-dire le but de la narration en général) qui a été racontée dans Le Chat botté était nouvelle dans l'histoire littéraire européenne, et un examen des formes narratives brèves au Moyen Âge démontre cette proposition. Prenons d'abord en considération les compilations des contes médiévaux. Dans Fairy Tales from Before Fairy Tales, Jan Ziolkowski discute de six contes qu'il qualifie de contes de fées. Quatre d'entre eux sont des "folktales», l'un d'eux est une légende religieuse, et l'autre, Asinarius, est un roman médiéval abrégé. Ce dernier ressemble quelque peu à un conte de fées moderne, mais il ne comporte pas ces composantes essentielles qui sont la souffrance du héros, son accomplissement de tâches et sa traversée d'obstacles. De même, dans Märchen des Mittelalters, Albert Wesselski a rapporté une grande variété de récits brefs médiévaux (Märchen), comprenant des contes urbains de voyouterie, comme ceux du Décaméron de Boccace, et des contes exemplaires racontés par des prêtres médiévaux depuis leur chaire d'église ${ }^{6}$. Il n'y a aucun conte de fées dont l'histoire se rapproche de celle du Chat botté (héros pauvre + magie + mariage à fortune). Certes, les lecteurs pourraient conclure que l'un des contes du $\mathrm{XIV}^{\mathrm{e}}$ siècle que Wesselski a pris des Novelle ${ }^{7}$ de Giovanni Sercambi (I348I424) est un conte de fées, parce qu'il raconte l'histoire d'un paysan dont les compagnons possèdent des attributs physiques extraordinaires qui vont l'aider à contracter un mariage royal. Mais, dans ce cas, le conte ne correspond pas à la catégorie du conte de fées pour des raisons historico-économiques : le personnage du jeune paysan appartient à une classe qui n'est plus depuis longtemps familière, celle de propriétaires terriens prospères, que les commerçants recherchaient souvent comme bons partis pour leurs filles. De là, la pauvreté du paysan de Sercambi n'a rien à voir

5. A. Jolles, Formes simples, Paris, Éditions du Seuil, 1972.

6. Le spécialiste très influent, Stith Thompson, a mentionné l'existence de recueils de contes exemplaires, mais il a bien précisé que ce n'était pas «le but de [son] étude de commencer une discussion des grands recueils littéraires de contes». Voir S. Thompson, The Folktale, Berkeley/ Londres, University of California Press, 1946, réimpr. 1977 et suiv. (ma traduction).

7. G. Sercambi, Novelle, Giovanni Sinicropi (éd.), Florence, Casa Editrice Le Lettere, «Filologia: Testi e studi, 5", I995. 
avec la misère noire que l'on peut trouver plus tard dans le conte de fées moderne.

Dans le troisième ouvrage, Formes médiévales du conte merveilleux, un trio de spécialistes distingués - Jacques Berlioz, Claude Brémond et Catherine Velay-Vallantin - font précéder le recueil d'un démenti significatif. Il est difficile, disent-ils, de définir ce qu'on entend par le terme conte merveilleux, et ils concluent que toute tentative en ce sens mènerait à une "discussion sans issue» (ouvr. cité, p. 9). Les éditeurs définissent le conte merveilleux médiéval comme une histoire qui comporte généralement un personnage royal parmi ses protagonistes, de nombreuses figures héroïques masculines plutôt que féminines, et un parent proche — père, mère, frère, sœur, mari, femme, fils, fille, belle-mère ou beaupère - qui joue un rôle décisif. Chacun des contes merveilleux de leurs recueils comporte un ou plusieurs éléments qui figurent dans les contes de fées modernes ${ }^{8}$. Mais, selon Wesselski, Berlioz, Brémond, Velay-Vallantin, Uther et les autres, l'observation qui s'impose, quand on lit les Märchen médiévaux et les contes merveilleux, c'est l'absence notable des contes de fées tant prisés dits "d'ascension", dans lesquels un pauvre héros ou une pauvre héroïne parvient à épouser, grâce à une intervention magique, un époux royal et devient ainsi riche.

La remarquable variété de contes qui circulent à l'époque du Moyen Âge chrétien reflète le brassage ethnique de l'Europe (celte, germanique et latin), ses cultures religieuses (païenne et chrétienne) et ses milieux sociaux (paysan, commerçant et noble). Développés, étendus et enrichis par les importations venues de l'est de la Méditerranée et des cultures asiatiques à travers Byzance à l'est et l'Espagne à l'ouest, ces contes étaient suffisamment variés pour plaire à chacun. Il y avait des contes paillards qui mêlaient la luxure débridée avec de l'humour de bas-étage, des allégories chastes qui récompensaient un désir fervent par une récompense divine, des interventions miraculeuses qui sauvaient les pécheurs et mettaient le diable en déroute et des farces urbaines qui se déroulaient dans des économies à rendement nul, où le gain d'une personne représentait la perte d'une autre. Ce sont des récits minimaux, des contes dont la brièveté se réduit à un début, un milieu et une fin, dénués de toute intrigue secondaire, de passages descriptifs ou encore de héros et hérö̈nes individualisés, tout ceci contrairement à ce qu'on trouve dans les autres formes littéraires.

8. Selon le catalogue Aarne Thompson Uther Tale-Type Index \#300-749. Voir H.-J. Uther, The Types of International Folktales: A Classification and Bibliography, 3 vols, Helsinki, Academia Scientiarum Fennica, 2004; Folklore Fellows Communication, p. 284. 


\section{Les premiers contes traitant du sujet de personnages royaux soumis à la souffrance}

Le Moyen Âge a produit une grande quantité de longs récits bien connus. Si l'on considère par exemple les innombrables romans arthuriens, les romans chevaleresques populaires comme Huon de Bordeaux ou Beuve de Hantone, des romances ressemblant aux romans telles Partonopeu de Blois, la légende en prose de Jean d'Arras intitulée Le Roman de Mélusine (1392-I393) et La Belle au cheveux d'or. Ces longs récits, et d'autres avec eux, font partie d'un corps de récits profanes richement variés qui ont survécu sous forme manuscrite depuis la fin du XII ${ }^{\mathrm{e}}$ siècle jusqu'au milieu $\mathrm{du} \mathrm{XV}^{\mathrm{e}}$, et même plus tard. Ils ont fait florès dans les premières décennies de la culture du livre, et ils ont fourni la matière aux intrigues des contes de fées ultérieurs dits de "restauration", ainsi que des motifs aux contes «d'ascension» comme à ceux de « restauration».

Mêlées à ces récits longs, se trouvent parfois des formes minimales qui dérivent des romances, des épopées et des nouvelles, mais qui ont abrégé leurs intrigues, les ont épurées de la plupart de leurs adjectifs et n'ont pas individualisé leurs héros et héroïnes souvent sans noms. Ces caractéristiques se brouillent avec les descriptions des contes de fées classiques comme le spécialiste suisse Max Lüthi l'a le premier montré9, mais les contes eux-mêmes racontent des histoires très différentes. Prenez par exemple le cycle de Sindibad (N.B. : il ne s'agit pas ici de Sindbad le marin). Il est entré en Europe sous le titre de De rege et septem sapientibus, mais il était souvent appelé le Dolopathos, d'après le nom du roi dans le récit-cadre. Les histoires du Dolopathos sont toutes des histoires exemplaires racontées pour mettre en garde un roi contre les actions inconsidérées, en l'occurrence l'exécution de son propre fils, que sa seconde femme a accusé d'agression sexuelle sur sa personne. Le jeune homme est innocent de ce crime, mais il a prononcé un vœu de silence qui le rend incapable de se défendre pendant plusieurs jours. Par conséquent, pour sauver sa vie, sept sages racontent au roi, son père, des contes exemplaires jusqu'à ce que le fils puisse parler à nouveau. Rassemblé par le moine cistercien Jean, à l'abbaye de Haute-Seille en Lorraine, à la fin des années Iıoo, le recueil original du Dolopathos contenait sept contes exemplaires et un conte magique (voir ci-dessous). Le conte magique et deux des contes exemplaires dérivent de sources orientales, ce qui fait de Jean

9. Voir son ouvrage de référence : The European Folktale: Form and Nature (1982). 
l'un des médiateurs de la matière narrative du Proche-Orient auprès des cercles littéraires européens du Moyen Âge ${ }^{\mathrm{I}}$. Les contes du Dolopathos étaient extrêmement populaires, particulièrement au $\mathrm{Xv}^{\mathrm{e}}$ siècle ${ }^{\mathrm{II}}$, et donc le seul conte magique qui s'y trouvait s'est trouvé lui aussi répandu au cours des décennies qui ont annoncé l'introduction de l'impression. De fait, son intrigue est pour nous d'une signification considérable. La voici :

Un jeune roi en train de chasser poursuit une biche jusqu'au fond des bois. Ayant perdu sa trace, il rencontre une nymphe qui tient une chaîne en or et qui se baigne dans une source. Il en fait sa femme (comme le texte nous le dit chastement), et celle-ci voit dans les étoiles qu'elle a conçu une fille et six garçons. La mère du roi, envieuse, feint de l'affection pour elle et ses sept nouveaux-nés, mais bientôt fait emporter les nourrissons et les remplace par des chiots. Sous l'influence de la bellemère, la reine se retrouve enterrée jusqu'au cou, nourrie de restes, éconduite par son mari et dédaignée de ses sujets, perdant peu à peu sa beauté à mesure qu'elle maigrit jusqu'à n'avoir que la peau sur les os.

Sept années plus tard, le roi aperçoit sept enfants munis de chaînes d'or autour du cou et se trouve touché par de l'affection naturelle ${ }^{\mathrm{r} 2}$. De retour au château, il dit à sa mère ce qu'il a vu, et celle-ci, à son tour, envoie une servante se renseigner. En observant les garçons se transformer en cygnes tandis que leur sœur tient leurs chaînes d'or, la servante vole les chaînes pour les donner à la reine. Sans leur chaînes, les frères sont incapables de retrouver forme humaine, et alors, avec leur sœur, ils s'envolent vers un étang près du château de leur père. Fasciné par leur beauté, le roi ordonne qu'on les protège. Pendant ce temps, leur sœur se présente au château chaque jour et mendie de la nourriture, qu'elle partage ensuite avec la reine à demi-enterrée sans savoir qu'il s'agit de sa mère. Le roi demande à connaître l'histoire de cette fille. Au cours du récit, la reine mère révèle sa culpabilité, la servante avoue sa complicité, et l'épouse ayant souffert pendant longtemps, retrouve sa place auprès du roi, et c'est la méchante mère qui se retrouve enterrée à sa place.

La transmission du Dolopathos s'est faite relativement lentement sous forme de manuscrit; mais avec l'invention de l'imprimerie, la cadence s'est accélérée, à mesure que la bibliothèque de colportage se répandait à travers l'Europe avec les tirages à la centaine. Finalement, la popularité du Dolopathos s'est étendue avec The Seven Wise Masters of Rome, et ses histoires se sont ainsi répandues en Europe à la fois sous forme manuscrite et sous forme de livres, jouissant d'une telle popularité que beaucoup d'éléments inhérents à l'unique conte merveilleux de Jean de Haute-Seille se sont mis à former partie intégrante des contes de fées dans les siècles qui ont suivi. Dans ce conte, les transformations qui s'opèrent entre cygne et

Io. W. Maaz, «Johannes de Alta Silva», col. 573.

II. Ibid., note 3 .

I2. Un exemple de la croyance médiévale selon laquelle on reconnaît son propre sang. 
humain relèvent de la magie bien que ce ne soit jamais annoncé comme tel. Les deux autres points communs que l'on peut constater entre le conte magique du Dolopathos et les contes de fées sont les suivants: l'hostilité violente de la belle-mère et la présence de l'or comme métal symbolique. Les sept années où la nymphe s'est trouvée enterrée vivante représentent la souffrance intense si caractéristique des contes de fées. Mais, étant donné que la victime est déjà mariée à un roi, il n'y a aucun besoin d'intervention magique pour réparer cette souffrance et être récompensé par un mariage avantageux. De même, il n'y a pas de mariage royal pour les autres enfants cygnes.

Un autre proto-conte de fées médiéval à signaler, c'est la nouvelle en vers $\mathrm{d}^{\prime}$ Asinarius $^{13}$. Écrite à peu près à la même époque où Jean de HauteSeille a rassemblé le Dolopathos, son intrigue, tout comme celle de la reine nymphe victimisée évoquée ci-dessus, relève de la restauration sociale :

Une reine longtemps stérile tombe enfin enceinte, mais au lieu d'accoucher d'un enfant humain, elle accouche d'un ânon. Élevé comme un prince, celui-ci compense son apparence répugnante par une grâce et une politesse exceptionnelles. Quand le prince entre dans le monde, il arrive à la cour d'un roi qui lui offre sa fille en mariage. Durant sa nuit de noces, Asinarius quitte sa peau d'âne tant détestée, mais il la retrouve vite au petit matin. Une servante qui observe ce phénomène étrange conseille au roi de dérober la peau d'âne et de la brûler. Ainsi privé de sa peau, Asinarius désire s'enfuir, jusqu'au moment où le roi lui promet de partager son royaume avec lui.

La féerie consciente est absente des 402 vers rimés d'Asinarius : la longue stérilité de sa mère est à attribuer à l'hostilité de Lucine, la déesse de la conception, et lorsqu'Asinarius naît sous forme d'âne, c'est bien un âne dont il s'agit. Après sa naissance, la mère suggère en toute lucidité qu'on noie son ânon de fils, mais le père plus respectueux intervient, en invoquant l'exemple des dieux païens des Romains comme Jupiter, Junon, Vénus et Hercule ${ }^{\mathrm{I}}$ pour justifier que le petit prince reste en vie, et parce qu'il est, après tout, l'héritier légitime du trône ${ }^{15}$. Les fées salvatrices ne faisaient pas encore partie du répertoire littéraire européen.

13. Albert Wesselski (1925) a estimé qu'Asinarius était le premier "Märchen» (Märchen des Mittelalters, p. 194). Le texte est disponible en traduction anglaise dans J. Ziolkowski, Fairy Tales from Before Fairy Tales (2006), p. 34I-350. Le texte latin peut également se trouver dans Asinarius, dans Commedie latine del XII e XIII secolo, S. Rizzardi (éd.), Gênes, Istituto di filologia classica e medieval, I983, t. 4, p. 137-25I.

I4. L. Harf-Lancner, Les Fées au Moyen Âge. Morgane et Mélusine : la naissance des fées, Paris, Champion, I984.

I5. J. Ziolkowski, ouvr. cité, p. 342. 
Même l'habileté remarquable qu'a le prince-âne à chanter d'une voix douce ne lui sert pas de compensation à son apparence horrible, un échange pourtant courant dans les contes modernes de féerie, où ordinairement le don d'une qualité accompagne et adoucit les effets d'un mauvais sort ${ }^{16}$. Au contraire, l'habileté de cet âne à charmer ses compagnons par ses douces mélodies est le résultat de beaucoup d'entraînement. De même, sa décision de quitter le royaume de son père est le résultat d'une réflexion raisonnée sur son sort, au cas où son père ne pourrait plus le protéger du mépris général. De même, le retrait de sa peau si détestée, au moment où il monte dans le lit nuptial, se fait tout simplement parce que c'est lui-même qui l'enlève pour se dévêtir, et la destruction de cette peau dans l'épisode suivant arrive elle aussi sans intervention féerique ni rédemption causée par une épouse charitable. Tous ces éléments font qu'Asinarius ne peut être considéré comme un conte de fées, particulièrement un conte de fées d'ascension de la sorte de ceux que Straparola publiera en $\mathrm{I}_{55 \mathrm{I}^{17}}$.

Comme beaucoup de nouvelles produites avant I45O, Asinarius utilise la sexualité comme un outil narratif et fait référence en particulier au plaisir féminin et au désir pour l'acte sexuel. Ainsi, dès le début, la reine mère avoue implicitement son penchant aux plaisirs nocturnes quand elle se plaint de ne plus pouvoir en jouir. Ensuite l'auteur d'Asinarius décrit le duvet pubien et la poitrine naissante de la princesse parce que ces signes physiques signifient clairement qu'elle désire ardemment prendre le premier homme qui se présentera à elle. Ensuite, l'offre que fait son père de la donner en mariage à Asinarius est enrobée de termes explicitement sexuels : "Désirez-vous que notre fille soit déposée dans vos bras pour qu'elle puisse participer à des jeux nocturnes avec vous?» Les plaisirs de la nuit de noces se traduisent par l'évocation de la vierge comme s'étant «ruée dans le lien conjugal». Ainsi le texte fait complaisamment la chronique de la nuit de noces :

16. Dans le Riquet à la houppe de Perrault, par exemple, le héros éminemment intelligent mais extrêmement laid a le don de donner l'intelligence à la femme qui l'aime. Des deux sœurs, la laide est intelligente et la belle stupide.

17. P. Gatti, «Elementi favolistici nell'Asinarius e nel Rapularius", dans La Favolistica Latina in distici elegiaci: Atti del convegno internazionale, Assisi 199o, G. Catanzaro and F. Santucci (éds), Assise, Accademia Properziana del Subasio Dentro Studi Poesia Latina in Distici Elegiaci, I99I, p. I49-160. Gatti place Asinarius dans la tradition des distiques élégiaques, qui ont transmis les contes ésopiques et autres contes animaliers du monde antique à travers le Moyen Âge et jusqu’à l'époque pré-moderne. Pour en savoir plus sur le rôle des distiques élégiaques dans l'histoire de la littérature médiévale, voir R. Adrados, «De la Fabula Griega a la Fabula Latina en Disticos Elegiacos" (I99I, p. 26-43), qui considère de tels distiques élégiaques comme une nouvelle phase dans l'évolution du "género fabulístico" (p. 27). 
Aussitôt il saute dans le lit et la fille aussi. Ce qui suit, ils le savent - et le lit aussi le sait. (Je ne pense pas non plus que l'homme qui se cachait pouvait ne pas avoir remarqué quels jeux se déroulaient ainsi pendant la nuit!) Il tempère la chaleur du désir amoureux pour un temps, et elle remplit les fonctions d'épouse pour son mari ${ }^{18}$.

Le profond sommeil dans lequel le couple tombe après avoir fait l'amour précipite la résolution de la nouvelle puisqu'il rend possible le fait que le père de la mariée dérobe et détruise la peau d'âne fatale.

À deux moments, le texte fait allusion au lectorat ou à l'auditoire visés. Ce sont des allusions légères sur lesquelles on pourrait passer facilement, mais elles laissent pourtant l'impression distincte qu'il s'agit de citadins aisés $^{19}$. Par exemple, l'âne refuse de s'asseoir au milieu des serviteurs ou parmi les chevaliers, prétextant qu'il n'est pas un âne d'étable ordinaire, mais qu'il vient de la noblesse urbaine. Avec cette remarque, nous réalisons aussi qu'Asinarius fait partie de ce riche trésor de contes urbains profanes qui, au Moyen Âge, opposaient les citadins raffinés aux rustiques paysans, les premiers ayant tendance à amplifier l'humour sur la difformité rurale de la peau d'âne. La seconde allusion apparaît avec la description de «la ville» décorée pour célébrer le mariage royal de l'âne, qui se trouve suivie d'une scène urbaine avec des spectacles acrobatiques donnés devant le peuple, et non confinés à l'intérieur des murs d'un château ${ }^{20}$.

L'auteur anonyme d'Asinarius n'a pas inventé cette histoire de zéro, mais il ne l'a pas non plus prise des Amours de Psyché et de Cupidon d'Apulée, où la monstruosité supposée de Cupidon n'est en fait pas réelle (alors que celle d'Asinarius l'est vraiment), mais est une laideur imaginaire insinuée par les sœurs jalouses de Psyché. Lauteur d'Asinarius, ayant reçu une éducation classique, connaissait certainement les Métamorphoses d'Apulée, communément appelé L'Âne d'or depuis des siècles, et il a certainement basé son titre dessus puisqu'il parle d'une «légende» qui lui a donné les faits. Mais le conte qu'il raconte est significativement différent.

La magie d'un âne qui accompagne sa douce voix à la cithare a fasciné Claude Brémond. Rappelons avec Brémond ces proverbes antiques : «Il

I8. J. Ziolkowski, ouvr. cité, p. 348.

I9. J. Ziolkowski avance que l'auteur d'Asinarius "a conçu ce poème pour un milieu scolaire» (p. 204, ma traduction), parce qu'il «explore des thèmes qui auraient trouvé résonance auprès de jeunes garçons lorsqu'ils les auraient parcourus» (p. 205, idem). Cela dit, mes propres lectures de textes scolaires de l'époque médiévale, pré-moderne et moderne (à la fois religieux et profanes) suggèrent en fait le contraire. En effet, il y a deux éléments essentiels dans les textes scolaires qui sont complètement absents d'Asinarius, à savoir d'une part les références explicites à l'enfance des lecteurs et d'autre part la prescription d'obéissance envers les plus âgés et les meilleurs que soi.

20. J. Ziolkowski, ouvr. cité, p. 347 et 348. 
n'existe aucun âne jouant de la guitare ni de taureau philosophe.» $\mathrm{Ou}$ encore : "Qu'est-ce que la voix d'un âne par comparaison à celle d'une alouette?» Ou encore : "Un âne ne prend aucun plaisir à la cithare.» Chacun de ces proverbes contribue à l'élaboration d'un sous-texte, ou d'un pré-texte, fondateur et humoristique, à Asinarius.

Brémond mettait en garde contre le fait de donner trop d'importance à Asinarius en tant qu'œuvre littéraire, mais cela n'empêche que ce conte a fait l'objet d'une large circulation à l'époque médiévale. Au XIII ${ }^{\mathrm{e}}$ siècle, dans le Registrum multorum auctorum, Hugo von Trimberg a ainsi classé Asinarius comme un livre qui se lisait dans les écoles aussi bien que dans les foyers ${ }^{21}$. Il nous paraît alors évident que Noël du Fail, au Xvi ${ }^{\mathrm{e}}$ siècle, fait référence à Asinarius dans son Propos rustique, quand il dit, sans d'autre explication, que l'histoire de Peau d'Âne a été racontée à l'occasion d'une petite réunion.

Asinarius est plein de dialogues intelligents, d'apartés amusants et d'embellissements érudits. Il pourrait, si ce n'était pour son contenu ouvertement sexuel, avoir été écrit par $\mathrm{M}^{\mathrm{me}} \mathrm{d}$ 'Aulnoy dans la France du XVII ${ }^{\mathrm{e}}$ siècle. En effet, le "Dauphin» de la conteuse est tout à fait du même esprit qu'Asinarius et comporte la même fin heureuse sous forme de restauration, même si le conte de $\mathrm{M}^{\mathrm{me}}$ d'Aulnoy tire d'une autre source, complètement différente, à savoir le Pietro Pazzo de Straparola. Toujours est-il qu'il paraît évident que l'auteur inconnu d'Asinarius était tout aussi jovial à la plume qu'Ovide avant lui et que Perrault et $\mathrm{M}^{\mathrm{me}} \mathrm{d}$ 'Aulnoy après lui.

\section{Les livres de miracles et les contes exemplaires}

Un second corpus d'histoires à mentionner, c'est le recueil espagnol, Disciplina clericalis, qui a émergé et a rapidement gagné en popularité dans le courant du XII ${ }^{\mathrm{e}}$ siècle. Composé par Petrus Alphonsus, un juif espagnol converti au catholicisme, il introduit des matériaux moresques, juifs et moyen-orientaux, dans la tradition narrative européenne. On a dit que Petrus Venerabilis de l'abbaye de Cluny, qui est mort en II56, avait rassemblé de la matière pour son propre livre : Liber de miraculis, pendant un voyage en Espagne en II42. Un autre moine, le cistercien Herbert de Clairvaux, a lui aussi publié un livre influent sur les miracles dans les

2. F. Wagner, «Asinarius», dans Enzyklopädie des Märchens, t. I, 1977, cols 865-867, ici col. 866.

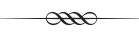


années $1178^{22}$. L'importance culturelle et littéraire des livres de miracles au $\mathrm{XII}^{\mathrm{e}}$ siècle repose principalement sur le fait qu'ils représentent une source d'images religieuses commune à un continent entier, que les compilateurs ont adoptées vers la fin du XII ${ }^{\mathrm{e}}$ siècle et au XIII ${ }^{\mathrm{e}}$ siècle en Allemagne ${ }^{23}$, en France $^{24}$ et en Italie ${ }^{25}$. De même, les chroniques continentales ${ }^{26}$ contenaient un bon nombre de contes qui pouvaient être mis en pratique par leur aspect exemplaire et propre à former une identité chrétienne parmi leurs auditeurs.

La plupart des livres de miracles ont été composés et disséminés en langue latine, mais certains ont été composés dans des langues parlées et comprises du peuple. Le Manuel des pechiez (c. I270) a été conçu pour un auditoire anglo-normand de langue française. Plus tard, Robert Mannyng l'a traduit pour les lecteurs de langue anglaise sous le titre de Handlyng synne. Dans un premier temps, les textes exemplaires ont été traduits ou publiés en espagnol, en anglais, en allemand, en français et en italien, et plus tard dans les langues périphériques de l'Europe comme l'islandais et le tchèque.

L'un des recueils de contes exemplaires les plus populaires de la période médiévale, la Scala coeli, a été compilé par le moine dominicain Johannes Gobi Junior (? - c. I350). Assemblé entre I323 et I330 dans un monastère de Provence, il était dédié à sainte Marie-Madeleine. Il faut savoir que les contes exemplaires de Johannes Gobi ont établi une norme qui a servi de modèle de la fin des années IIoo jusqu'au début des années I300. De là, en effet, sont apparus les genres de contes que l'on trouve assemblés dans la Scala coeli, aussi bien que les contes individuels eux-mêmes. Ils ont donc eu une résonance large et qui a duré.

Le monde des contes exemplaires du Moyen Âge est un monde dans lequel ce qui est étrange à rapporter relève plus du miracle que de la magie. C'est un monde dans lequel les figures divines interviennent couramment dans la vie quotidienne pour sauver des pécheurs repentants. Le grand

22. Voir M. J. Lacarra, Cuentistica medieval en Espana: Los origenes (Saragosse, Departamento de literature Espanola, 1979) et Cuentos de la edad media (Madrid, Editorial Castalia, coll. "Odres nuevos", 1986), ainsi que R. E. Marsan, Itinéraire espagnol du conte médieval VIII'-XV siècles (Paris, Klincksieck, 1974).

23. Caesarius of Heisterbach, Dialogus miraculorum (I219-I223) et Libri VIII miraculorum (I225-I226).

24. Le dominicain Guillaume Perraud, Summa de vitiis et virtutibus (before I250).

25. Giovanni di San Geminiano, Summa de exemplis et similitudinibus rerum (c. 1300).

26. Par exemple, la chronique du cistercien Hélinand de Froidmont (début du XIII ${ }^{\mathrm{e}}$ siècle), le Speculum historiale du dominicain Vincent de Beauvais, et bien plus tard la Chronica du franciscain Salimbene (c. I290). 
nombre de manuscrits de recueils de contes exemplaires prouve leur large distribution et leur usage courant au Moyen Âge. À la bibliothèque de l'université de Liège, par exemple, il y a environ 2000 manuscrits de contes exemplaires, produits à l'origine par les frères de Sainte-Croix. Des recueils de contes exemplaires figurent aussi beaucoup parmi les premiers livres imprimés. Les prêcheurs médiévaux ont répandu les motifs et les événements miraculeux des collections de contes exemplaires pendant le haut Moyen Âge et la fin du Moyen Âge, leur résonance populaire étant attestée par leur présence à la fois dans les recueils de sermons du début du XIII ${ }^{\mathrm{e}}$ siècle, comme ceux de Jacques de Vitry et de son contemporain Odo of Cheriton.

Les recueils de contes exemplaires ont été organisés de la manière suivante pour faciliter la documentation et l'usage pratique : les histoires ont été classées par ordre thématique et alphabétique pour inciter plus facilement à un embellissement ou à un récit tout en couleurs. Comme chaque recueil contenait plus de Iooo histoires, une telle organisation était nécessaire.

Tous ces recueils ont été copiés à la main à la douzaine, quelquefois même à la centaine. Les recueils étaient physiquement transportés par des moines mendiants qui voyageaient à pied, qui dormaient sur la même paillasse que leurs hôtes et qui mangeaient à la table de gens humbles. Ces livres, qui se présentaient sous forme de petits abrégés portables, ont beaucoup enduré. Couverts d'annotations, tachés par la sueur, froissés et déchirés par un usage constant, ils ont perdu de leurs pages, les pages les plus utilisées étant souvent parties les premières ${ }^{27}$. Le fait que tant d'exemplaires de manuscrits aient survécu, suggère que le nombre de recueils de contes exemplaires sous forme manuscrite, en circulation à cette époque, a bien surpassé ceux qui se trouvent maintenant dans les bibliothèques et les archives ${ }^{28}$.

Examinons par exemple la Scala coeli de Johannes Gobi Junior en détail $^{29}$. Chaque histoire est structurée selon le modèle depuis longtemps établi pour les contes exemplaires : d'abord l'annonce de l'enseignement de l'histoire, ensuite l'histoire elle-même et enfin une répétition de l'ex-

27. Ceci est tout aussi évident dans les manuscrits médiévaux qui tous ont été malmenés, comme par exemple l'Historia Scholastica de Petrus Comestor. Voir R. Bottigheimer, "Sacred Stories, Eternal Words, and Holy Pictures,» dans Exhibit Catalog (typescript), Houghton Library, Harvard University.

28. Pour ma discussion sur les livres de miracles, je reconnais ma dette envers Berlioz, Brémond et Velay-Vallantin dans Formes médiévales du conte merveilleux.

29. Voir l'édition critique de M.-A. Polo de Beaulieu (éd.), La Scala coeli de Johannes Gobi [c. I323-I330], Paris, Éditions du CNRS, I99I. 
plication du message introducteur. Les histoires elles-mêmes étaient arrangées par catégories selon le sujet (depuis «Abstinence» à "Usure»), aussi bien que par catégorie selon l'auditoire (par exemple "Marchand», "Chevalier», "Épouse» et "Mari»).

L'intérêt de la Scala coeli pour les historiens du conte de fées repose sur le fait que les gens du peuple aimaient écouter les sermons qui étaient ornés d'histoires. Il est tentant pour les spécialistes de l'oralité de conclure que si l'on retrouve plus tard dans la tradition orale la présence de motifs caractéristiques à la Scala coeli, c'est donc que la tradition orale est à l'origine de ce texte. Pourtant, on ne peut pas dire que la Scala coeli dérive de la tradition orale, étant donné que seulement cinq de ses récits disent que leur compilateur les a "entendu(e)s", et que, sur ces cinq, quatre sont à attribuer non au folklore collectif ou à un paysan individuel, mais à un homme saint ou à un prêcheur. Le seul conte «entendu» qui n'est pas à attribuer à une source religieuse, le $\mathrm{n}^{\circ} 686$, raconte une intervention miraculeuse de la Vierge Marie qui a été plus tard documentée comme Miracle $\mathrm{n}^{\circ} 22$ dans un recueil de miracles de la Vierge datant de I434, compilé par Jean Herolt ${ }^{30}$ (mort en I468). Il semble plutôt que la Scala coeli ait disséminé largement toute une quantité de sources antiques ${ }^{31}$ et récentes ${ }^{32}$ que l'érudit Johannes a fait figurer dans la préface ${ }^{33}$. Ainsi le codex de la Scala coeli est devenu une vaste source de connaissances populaires de contes exemplaires ésopiques et magiques, relayant ces histoires vers l'auditoire des sermons en Europe. Plus tard, la machine à imprimer a facilité la transmission des histoires de Johannes Gobi dans le monde pré-moderne, où elles ont continué à proliférer ${ }^{34}$.

30. Pour en savoir plus sur les croisements entre recueils, voir G. Philippart et B. Dunn-Lardeau, «Les miracles de Jean Herolt (I434) et la Légende dorée», Le Moyen Français, n³2, 1993, p. 533-567. 3I. Par exemple, Johannes Gobi Junior a extrait des histoires profanes d'Ésope, de Macrobe, d'Hermès Trismégiste, d'Ovide, de Sénèque, de Suétone, de l'Historia Troiana et de l'Historia Antiqua, de même que certains des écrits de théologiens chrétiens de l'Antiquité.

32. Les sources les plus importantes de Johannes Gobi Junior pour l'histoire des contes populaires brefs qui sont sous-jacents dans les contes de fées comprennent la Gesta Romanorum, Liber de Septem Sapientibus, Miracula Beate Virginis, Alphabetum Narrationum (c. 1308-1310), et plusieurs autres recueils de contes exemplaires, tels que la Gesta Alexandri et Barlaam.

33. Rudolf Schenda attire l'attention sur l'importance des ordres religieux auxquels les prêcheurs appartenaient, en ce que cela a influé sur la manière dont les récits se sont conservés et se sont répandus de 500 à 1500 , et il nomme en particulier les prêcheurs suivants : Jacques de Vitry (II60/70-I240), Caesarius of Heisterbach (c. II80-I240), Étienne de Bourbon (XIII ${ }^{\mathrm{e}}$ siècle) et John Bromyard (XIv siècle). Voir R. Schenda, Von Mund zu Ohr (Göttingen, Vandenhoeck \& Ruprecht, 1993), p. I4I.

34. Des versions imprimées de la Scala coeli ont été répertoriées dans les Allemagnes (Lübeck, I476; Ulm, I480; Strasbourg, I483), les Pays-Bas (Louvain, I485) et l'Angleterre (Boston, I505). Voir aussi G. Huet, «Les Rédactions de la Scala coeli» (1920). Les imprimeurs apparemment en ont 
En ce qui concerne la survie des écrits du monde antique dans les contes de fées de l'époque pré-moderne, il faut noter que Johannes Gobi utilisait, entre autres, les écrits d'Ovide. Ce fait signifie que c'est bien lui qui a transmis l'imagerie ovidienne du monde antique méditerranéen au monde médiéval européen et que les prêcheurs qui ont utilisé les contes du recueil ont fait la même chose. Par conséquent, chaque fois qu'un prêcheur itinérant racontait dans son sermon l'une des histoires de Johannes Gobi dérivée d'Ovide, il propageait ce conte auprès de 20-IOO auditeurs, fonctionnant ainsi comme un pont littéraire entre le monde antique et le monde médiéval.

Le grand nombre de contes de la Scala coeli classés comme contes magiques dans l'ATU 300-749, dans le recueil des Grimm, rend évident le fait que les recueils de contes exemplaires ont fonctionné comme un agent de génération dans l'histoire des contes merveilleux ${ }^{35}$. La puissance narrative de la Scala coeli est aussi évidente par le grand nombre de ses contes qui ont émergé à nouveau en tant que «folktales» dans les siècles ultérieurs ${ }^{36}$. Mais d'une manière générale, on peut dire que les spécialistes du conte de fées n'ont pas exploré les recueils de contes exemplaires en profondeur ${ }^{37}$.

modifié le contenu en enlevant I30 des I ooo contes exemplaires. Ainsi l'histoire du contenu de la Scala coeli suggère fortement que les goûts avaient changé, et que ces modifications ont pris place en conjonction avec l'innovation technologique. L'aspect technologique est d'une importance centrale ici car l'invention de la machine à imprimer a grandement étendu l'exposition du public aux histoires de Johannes Gobi. On ne peut pas savoir si ces changements faits au contenu de la Scala coeli représentent l'évaluation que l'éditeur a faite du goût du public, l'imposition par la censure de critères nouvellement formulés de moralité publique, ou bien l'expression réelle ou reportée du choix personnel du lecteur et la preuve de son pouvoir d'achat. Tout ce que nous savons, c'est que I3O contes ont été omis. Une analyse et une caractérisation de la nature des contes omis auraient le potentiel de révéler précisément ce qui était tombé en défaveur entre 1476 et 1505 .

35. \#64 = ATU 516 Amicus und Amelius (adjuvant surnaturel); \#72 = ATU 325 Magician and his Pupil (objets magiques); \#180 = ATU 706 Maiden without Hands (via l'Historia Regum Franciae); \#184 = ATU 712 Crescentia (femme calomniée); \#313 = ATU 510B Peau d'Asne (inceste entre père et fille); \#342 = ATU 592 Dance among Thorns (objets magiques); \#474 = ATU 505 The Grateful Dead (adjuvant surnaturel); \#520 = Motif K 2III Joseph and Potiphar's wife (via Liber Septem Sapientum, avec son roi "Dyocletianus»); \#520 = ATU 67I The Three Languages (puissances surnaturelles); \#530 = ATU 613 The Two Travelers (magic objects); \#537 = ATU 654 The Four Skillful Brothers (puissances surnaturelles); \#538 = ATU 55I Water of Life (aide surnaturelle); \#556 = ATU 47IA The Monk and the Bird; \#618 = ATU 655 The Wise Brothers (puissances surnaturelles); \#756 = ATU 470A The Offended Skull; \#797 = ATU 470B The Land Where No One Dies.

36. Voir la liste dans M.-A. Polo de Beaulieu, «Johannes Gobi Junior», Enzyklopädie des Märchens, t. 7, 1993, cols 598-600.

37. Christoph Daxelmüller renforce cette idée en 2006 dans son compte rendu de Teufelsmacht und Hexenwerk de U. Brunold-Bigler (2003). Voir C. Daxelmüller, "Ursula Brunold-Bigler: Teufelsmacht und Hexenwerk. Lehrmeinungen und Exempel in der Magiologia des Bartholomäus 
Le recueil de Johannes Gobi a été l'un des recueils de contes exemplaires parmi bien d'autres qui a répandu des intrigues contenant le genre d'éléments magiques parvenus ensuite à caractériser les contes de fées à l'époque moderne. La Legenda Aurea (c. I260) de Jacobus de Voragine, sans doute le livre le plus lu dans l'Europe du Moyen Âge, que ce soit sous forme manuscrite ou imprimée, a répandu des contes sur des tueurs de dragons aussi bien que sur toute une variété d'événements miraculeux qui ont été perçus dans les siècles ultérieurs comme des manifestations magiques ${ }^{38}$. L'influence des histoires de la Scala coeli de Johannes Gobi est incommensurable. Par exemple, un manuscrit islandais de la fin du Moyen Âge comprend l'histoire $\mathrm{n}^{\circ}$ 618 de la Scala coeli ${ }^{39}$. Une autre histoire de la Scala coeli (la $\mathrm{n}^{\circ} 538$ ) a continué à être utilisée en extraits dans des recueils d'histoires profanes ou religieuses jusque dans le courant du $\mathrm{XVII}^{\mathrm{e}}$ siècle, son contenu entretenant une étroite relation avec l'histoire des contes de fées européens ${ }^{40}$ :

Un roi malade qui ne peut être guéri que par de l'eau provenant de la fontaine de la vie (aqua fontis viventis) promet son royaume à quiconque lui apportera de cette eau aux vertus restauratrices. Son premier fils cherche sur les berges des fleuves ( $p e r$ riparias), le second dans les plaines (per planicies), et le troisième dans les montagnes (per montes). Un vieil homme dirige ce dernier vers la fontaine de jouvence (fons juventutis). Pour l'atteindre il doit passer devant un serpent mortellement dangereux, un groupe de jeunes filles choristes, des chevaliers et des barons armés (épreuves), avant de pouvoir essayer d'ouvrir en silence le portail tout recouvert de clochettes d'un palais (tâche impossible). À l'intérieur du palais, une fille tenant la clé de la fontaine de jouvence lui dit qu'il doit l'épouser, ce qu'il fait. De retour chez lui, il reçoit le royaume de son père ${ }^{4 t}$.

Dans cette histoire, la quête dangereuse d'une substance mythique (l'eau de la vie) et protégée par toute une série d'épreuves est une tâche impossible. Un vieil homme non identifié donne au héros des informations importantes, mais le héros, non secondé par une aide extra-terrestre ou

Anhorn (I6I6-1700), Chur, Kommissionsverlag Desertina, (Quellen und Forschungen zur Bündner Geschichte, I2), 2003", Fabula, vol. 47, n³/4, 2006, p. 324-327, ici p. 323.

38. B. Fleith et A. Wenzel, «Legenda aurea», dans Enzyklopädie des Märchens, t. 8, 1996, cols 846855 (1996), ici col. 846. Voir cols 85i-852 pour le conte-type et la liste des motifs.

39. J. Berlioz et al. (éds), Formes médiévales du conte merveilleux, ouvr. cité, p. 89.

40. Ceci est pris d'une traduction en français dans ibid., p. 99-Ior. Le latin original se trouve dans M.-A. Polo de Beaulieu, éd. de 1991, p. 399-400.

4I. L'explication ouverte que la fille fait pour justifier pourquoi le jeune prince doit l'épouser donne un bon exemple du style sec de cette histoire: "Dictum est mibi a domine patre meo quod cum illo milite contraham si, habita victoria de omnibus impedimentis sibi objectis in via, ad me salvus pervenerit. Et quia tu es elle, non solum de fonte juventutis habebis sed me habebis in sponsam." (M.-A. Polo de Beaulieu, ouvr. cité, p. 399.) 
magique, réussit à relever le défi grâce à ses vertus innées, son courage et son obéissance. En ce qui concerne la distinction entre le magique et le miraculeux, une explication rhétorique très explicite en trois parties, qui constitue le dernier tiers du récit, délimite le contenu religieux du conte. Sur le plan spirituel, le texte de Johannes Gobi Junior nous dit que les trois fils humains d'un père malade représentent trois sortes de personnes. La première recherche les plaisirs (delicias) et fréquente les côtes. La seconde cherche les plaisirs (à nouveau delicias) dans les plaines, le lieu biblique traditionnel du péché. La troisième, qui passe par la pénitence (penitencias) et choisit de prendre les routes de montagne, passe par quatre épreuves (quattuor impedimenta) : le goût du serpent pour la vengeance (affectio vindicte qui est coluber); les plaisirs de la chair représentés par les femmes (carnis complacentie que sunt mulieres); l'attachement des chevaliers pour les choses terrestres et la peur de la pauvreté (affectio rei terrene et timor paupertatis qui sunt milites); et un appétit de reconnaissance et d'élévation représentés par les cloches de l'histoire (appetitus honoris qui sunt campanule). Le fils qui est victorieux utilise des moyens matériels et spirituels pour affronter les épreuves qui lui sont assignées : le bâton de pèlerin qui rappelle la Passion du Christ, la fuite dans la pauvreté, la récompense céleste espérée et l'«éponge de l'humilité». Seul le troisième et dernier fils est capable de pénétrer dans le palais de la grâce, de trouver l'amour spirituel et d'obtenir l'eau de la rémission des péchés, de même que la jeune femme à l'intérieur du palais qui devient son épouse ${ }^{42}$.

Le commentaire du prêcheur allonge le conte de moitié, dénaturant ainsi l'histoire et lui ôtant toute sa validité narrative. Comme Johannes Gobi le raconte dans son intégralité, ce n'est pas un conte de fées, même s'il contient en substance un assemblage de motifs significatifs caractéristiques des contes de fées d'ascension et de restauration à partir du $\mathrm{XVI}^{\mathrm{e}}$ siècle - le plus jeune des trois frères, la quête, les épreuves et la tâche impossible, l'accomplissement victorieux qui lui vaut une épouse royale et l'objet de sa quête, l'eau de la vie. La manière dont laquelle Johannes Gobi fait fonctionner ces motifs dans son conte, néanmoins, ne produit pas un conte de fées, mais un conte religieux qui manifeste une vision médiévale du monde chrétien. S'il s'agissait d'un conte de restauration, soit son héros souffrirait de la difficulté à surmonter les tâches et les épreuves qui lui sont présentées, soit sa bonté inaltérable appellerait à un concours magique sous la forme d'un adjuvant pour qu'il puisse accomplir ses tâches. Mais

42. J. Berlioz et al., ouvr. cité, p. IOO-IOI; M.-A. Polo de Beaulieu, ouvr. cité, p. 399-400. 
à aucun moment où l'histoire est racontée et interprétée, ces éléments n'apparaissent d'une manière qui corresponde à un paradigme de conte de fées.

La littérature exemplaire du Moyen Âge nous transporte dans un étrange état de conscience et d'identité. Poursuivons avec un autre conte exemplaire du haut Moyen Âge, et dont l'intrigue aussi bien que l'issue confondent le lecteur moderne :

Une fille non encore en âge de se marier ${ }^{43}$ a été promise à un chevalier. Quand il part outre-mer, il la laisse ainsi que son royaume sous la protection de son sénéchal. Pendant son absence, la fille devient une charmante jeune femme, et le sénéchal lubrique la viole. Quand celui-ci s'endort, la jeune femme profondément déshonorée l'étrangle et demande à un compatriote se trouvant à la cour de l'aider à se débarrasser du corps. Mais, la traitant de meurtrière, il refuse de l'aider à moins qu'elle ne couche avec lui. Alors, elle promet de le faire («avec sa bouche, mais pas avec son cœur»), mais quand il transporte le corps dans un sac jusqu'à un pont, elle le pousse à l'eau, lui et son fardeau, et il se noie. Quand le chevalier revient chez lui, sa protégée lui cache la perte de sa virginité en faisant glisser subrepticement dans les draps du lit nuptial une servante vierge. Une fois déflorée, cependant, la servante refuse de partir, alors l'héroïne l'étouffe, camoufle son corps dans un coffre auquel elle met le feu, réveille son mari, et s'enfuit avec lui dans un endroit sûr.

Longtemps après, la jeune femme avoue ses méfaits à son confesseur, qui lui prescrit une pénitence journalière (porter la haire) et une autre hebdomadaire (se mettre au pain et à l'eau). Mais le diable finit par inciter le confesseur à la convoiter. Menaçant de révéler l'histoire à son mari, il en exige de se soumettre sexuellement à lui. Confiante en la miséricorde divine, elle refuse. [Sa confiance en Dieu est le point pivotal de l'histoire et c'est apparemment la raison pour laquelle les prêcheurs du Moyen Âge racontaient cette histoire problématique.] Le confesseur révèle donc l'histoire au mari (sauf bien sûr sa propre action d'extorsion), garantissant son propos en offrant ses yeux si ses accusations venaient à être montrées fausses.

Le vendredi suivant, le mari et le confesseur se rencontrent dans la chambre de la femme, mais Dieu a enlevé tous les signes de sa pénitence pour ses mauvaises actions passées, transformant sa haire en une chemise de lin doux et l'eau en vin. Le chevalier transperce alors les yeux du confesseur.

Les derniers mots de l'histoire renforcent sa morale : «Et ainsi Dieu sauva la femme repentante qui avait renoncé au péché et, dans sa grande miséricorde, la soulagea de sa culpabilité ${ }^{44}$.»

43. "Ein noch nicht mannbares Mägdlein» est la traduction de A. Wesselski dans Märchen des Mittelalters (1925), p. 46.

44. A. Wesselski, Märchen des Mittelalters, 193I, p. 46-47. A. Wesselski a tiré cette histoire de la Compilatio singularis exemplorum reproduite chez Alfons Hilka. A. Wesselski a aussi cité Reinhold Köhler, Kleinere Schriften, J. Bolte (éd.), I898 f., t. 2, p. 303-399; V. Chauvin, Bibliographie des ouvrages arabes ou relatifs aux arabes..., p. 217 ff.; A. L. Jellinek, Euphorion, 9, p. I63; J. Bolte 
La triple meurtrière, dans l'histoire allemande correspondante, était décrite seulement deux fois : d'abord dans sa condition pré-pubertaire (noch nicht mannbar) et à la fin en tant que pénitente et réformée (bereuende und der Sünde entsagende $\left.{ }^{45}\right)$. Ce conte n'est pas à propos du meurtre et de ses conséquences, mais à propos de la divine récompense pour la pénitence.

Au Moyen Âge, les contes exemplaires faisaient partie de la vie religieuse. À l'église, ils étaient intégrés dans les sermons, et ils étaient aussi récités oralement dehors en plein air. Les légendes mariales appartenaient davantage au monde des femmes, peut-être comme lecture de divertissement pour les privilégiées ou comme consolation pour celles qui étaient plus défavorisées. Comme autre objet de divertissement, il y avait aussi les recueils de contes profanes, tels que le Decameron de Boccace. Les écoliers quant à eux s'entraînaient à la lecture avec des livres tels qu'Asinarius, I Reali di Francia et le Ovidius Moralizatus, qui proposaient des exemples de bons comportements en y mêlant l'aventure.

\section{Les légendes mariales}

De la fin du XII ${ }^{\mathrm{e}}$ siècle au milieu du Xve siècle, les contes profanes et les fabliaux sont restés plus ou moins inchangés en ce qui concerne leurs descriptions de l'indépendance des femmes et de la manière dont celles-ci se protégeaient des agressions sexuelles. Vers le début de cette période, les contes religieux, y compris les contes exemplaires, racontaient le même type d'histoire. Cela n'a pas été mieux illustré que dans les légendes mariales. Ciblées principalement autour des femmes, les légendes mariales valorisent leurs héroïnes et compatissent avec leur souffrance. Les difficultés des

et G. Polivka, Anmerkungen zu den Kinder- und Hausmärchen der Brüder Grimm, 1913 f., t. 3, p. $449 \mathrm{ff}$. De même, le Catalogue of Romances in the Department of Manuscripts in the British Museum (le tome 3 a été édité par J. A. Herbert en I910), t. 3, p. 563 (\#46); et A. Bricteux, Contes persans, p. $38 \mathrm{ff}$. E. Tegethoff a retravaillé le texte de Méons dans le t. I, p. Io9 ff. (Toutes les citations sont de Wesselski, Märchen des Mittelalters [p. 208-209].)

45. A. Wesselski, Märchen des Mittelalters, 1925, p. 47. Si l'on veut une mesure objective pour les contes minimaux, on pourrait pour chaque conte établir un index basé sur la proportion des verbes par rapport au nombre de mots en général, et on trouverait alors que cela oscille entre $10 \%$ et $15 \%$. C'est une proportion courante dans les textes qui sont racontés oralement ou représentés sur scène, et pas seulement dans les récits minimaux. Par exemple, une romance typique, comme Der arme Heinrich, qui est contemporaine aux textes dont nous parlons, contient une proportion de verbes de $13 \%$ par rapport au nombre total de mots, et une épopée telle que Nibelungenlied en contient une proportion de $12 \%$, alors qu'un article encyclopédique ou un traité philosophique en contient une proportion bien plus basse. 
femmes dans ces histoires sont généralement causées par des parents de sexe masculin ou des hommes d'église abusifs, comme le montre l'exemple du confesseur de la jeune femme que nous avons décrit plus haut. Dans le conte suivant, la Vierge Marie procure une couverture divine pour un inceste maternel et un infanticide :

Un vieux sénateur romain est marié à une jeune et belle femme, vertueuse de surcroît. Ils restent longtemps sans enfants, mais peu avant la mort du sénateur, sa femme accouche d'un fils. Dans la solitude de son veuvage, la femme du sénateur prend le bébé dans son lit et partage ainsi sa couche avec lui jusqu'à l'adolescence de ce dernier, où une nuit, malgré lui il la met enceinte. Quand elle réalise qu'elle a conçu un enfant de son propre fils, la veuve envoie ce dernier au loin pour le protéger de la connaissance du péché qu'il a commis. Quand l'enfant naît, elle le tue et l'enterre en secret.

Rongée par la culpabilité, elle se rend à Rome déguisée en Maître d'art. Intelligente et sage, elle devient une conseillère de confiance de l'empereur. Mais un jour le diable révèle ses péchés passés au pape, lui offrant comme preuve de lui montrer les os cachés de son enfant assassiné. La "conseillère» nie l'accusation et se jette à la merci de la Vierge Marie, lui promettant de lui construire une chapelle si elle peut empêcher la découverte et l'exécution. La Vierge Marie accepte et enlève les os de l'enfant de sa tombe secrète. Le diable creuse en vain et la veuve rentre chez elle et construit la chapelle.

Ceci n'est pas un scénario isolé, mais plutôt un scénario typique du genre médiéval éphémère des miracles mariaux qui montre Marie aidant les femmes en détresse. Un simple aperçu des légendes mariales mène à la conclusion qu'elles ont moins à voir avec la morale qu'avec le dessein d'établir la suprématie de Marie sur le diable dans l'esprit des femmes qui souffrent.

\section{Les chroniques médiévales, les romances de cour et les contes de fées}

Les chroniques médiévales étaient censées transmettre des comptes rendus historiques d'événements passés. La forme de la chronique pouvait aussi être utilisée comme structure pour les romances de cour, comme l'a dit Andrea da Barberino (c. I370-c. I43I), quand il a composé I Reali di Francia. Caractéristique d'un changement par rapport à l'orthodoxie traditionnelle des textes exemplaires médiévaux et des légendes mariales selon lesquelles les figures divines de Dieu et de la Vierge Marie ont contrôlé la direction de l'histoire de l'humanité, notre auteur a incorporé dans ses contes des improbabilités et des tournures d'événements inattendues sans aucune référence à l'entremise divine. C'est surtout le cas dans l'étrange histoire 
de la vie de Berthe, la mère de Charlemagne, dans le Livre du Reali di Francia.

Fille du roi hongrois Floire et de la reine Blancheflor ${ }^{46}$, la beauté parfaite de Berthe est pourtant gâchée par ses grands pieds. Cette difformité n'empêche pas le roi mérovingien Pépin de la demander en mariage. La nourrice de Berthe, Margiste, lui dit que Pépin a l'intention de la tuer pendant la nuit de noces afin de lui substituer sa propre fille, Aliste, dans le lit conjugal. Le lendemain Aliste-Berthe dit à Pépin que Berthe-Aliste a l'intention de la tuer afin que la vraie mariée soit exécutée. Pépin fait alors emmener la vraie Berthe dans les bois pour être tuée, et Margiste demande à voir le cœur de Berthe comme preuve de sa mort. Des serviteurs secourables lui substituent le cœur d'un sanglier après avoir abandonné Berthe dans la forêt, où celle-ci trouve refuge dans la chaumière d'un couple de paysans.

Quand la mère de Berthe vient rendre visite à sa fille adorée, elle reconnaît que la femme de Pépin est un imposteur (aux pieds de taille normale). La trahison de Margiste est ainsi révélée et celle-ci est condamnée à mort, tandis que sa fille, la fausse mariée, est envoyée dans un couvent.

Pépin rencontre Berthe sans la reconnaître, et tombe amoureux d'elle. Il omet de s'identifier et l' "épouse». La mère de Berthe, dans un second voyage depuis la Hongrie jusqu'à la France, reconnaît sa fille et fait en sorte que son statut royal soit restauré après toutes ces années de souffrance.

Il faut noter que cette chronique mérovingienne, théoriquement historique, a été composée à la fin du XIv ${ }^{\mathrm{e}}$ siècle, c'est-à-dire quelque huit cents ans après les événements supposés, et doit son origine non à des documents historiques mais au contenu de chansons de geste françaises, et peut-être aussi à un des Lais de Marie de France. Écrit en italien, I Reali di Francia est devenu une source narrative médiévale et pré-moderne de laquelle les auteurs ultérieurs de romances et d'épopées ont tiré beaucoup et souvent. Par conséquent, bon nombre de motifs et d'épisodes de la chronique de la vie de Berthe font partie de l'héritage du conte de fées européen :

\footnotetext{
46. Les noms de ce couple ont été clairement empruntés aux amants illustres Floire et Blancheflor, dont l'histoire de la dévotion mutuelle qui remonte à l'enfance a été suivie d'une loyauté à l'adolescence et couronnée à l'âge adulte par l'amour. Répertoriée partout en Europe - en France ( $c$. II70 et après), en Allemagne ( $c$. I200 et après), en Angleterre ( $c$. I250), en Norvège ( $c$. I300), en Italie (dans les années I300), en Grèce (fin des années I300 - début des années I400), au Danemark (I400) et en Espagne (I5I2) - l'histoire de Floire et de Blancheflor n'a aucun rapport avec celle de Berthe. Pour plus de renseignements, voir E. Frenzel, «Floire et Blancheflor ", Enzyklopädie des Märchens, t. 4, 1984, cols I310-1315.
} 
I. Une fausse mariée remplace la vraie mariée;

2. La vraie mariée est condamnée à mort, mais est épargnée par des serviteurs charitables (un motif maintenant inséparable de la BlancheNeige des Grimm);

3. Le cœur d'un animal sauvage, qui dans les contes ultérieurs a pu être remplacé par son foie ou ses poumons, est présenté comme fausse preuve de la mort de l'héroïne;

4. L'héroïne trouve refuge chez des habitants des bois, un motif qui de même fait partie de plusieurs contes de Grimm;

5. Un roi tombe amoureux d'une belle jeune fille qu'il rencontre en chassant dans une forêt (ce qu'on trouve dans la Grisélidis de Perrault aussi bien que dans plusieurs contes de Grimm);

6. Le roi ne s'identifie pas, mais il couche avec l'héroïne (ce motif a disparu au cours du XVIII ${ }^{\mathrm{e}}$ siècle, moralement plus conscient, et ne figure pas dans le recueil des Grimm au XIx ${ }^{\mathrm{e}}$ siècle);

7. L'héroïne finit par être identifiée comme la véritable épouse et l'histoire se conclut ${ }^{47}$.

Depuis sa première composition existante sous forme de manuscrit, le conte de Berthe est passé dans les autres langues, ce qui a produit un motif basique et dense qui s'est répandu à travers l'Europe. En outre, ce motif mis en place était si riche qu'il a pu être récrit selon les normes propres à différents genres littéraires médiévaux de chaque pays (ou de chaque langue) dans lesquels il était remanié. En France, par exemple, l'essentiel du conte de Berthe a été intégré à l'épopée chevaleresque Valentine et Orson, sans conteste le morceau de littérature populaire le plus influent qui ait jamais été composé, avec des centaines de manuscrits et tout autant d'imprimés, en allemand, en français, en anglais et en italien. I Reali di Francia a aussi été l'un des premiers livres imprimés d'Italie, avec des éditions qu'on fait remonter à I49I, I5II, I5I6 et I547. En dehors de l'Italie, il a circulé dans le nord ainsi que dans le sud de la France, sous forme de manuscrits intitulés respectivement Berte aus grans pies et Berta da li pe grandi ${ }^{8}$.

Le fait que la bigamie ait existé parmi les ancêtres mérovingiens de Charlemagne est indiscutable, de même que les persécutions des différentes reines Berthe ayant réellement existé. Néanmoins, la création littéraire du

47. M. Rumpf explore de tels motifs dans «Berta» (1979), Enzyklopädie des Märchens, t. 2, 1979, cols I57-I58.

48. A. Adler, "The Structural Meaning of Berta da li pe grandi », Italica, I950, p. IOI. 
XIV $^{\mathrm{e}}$ siècle qu'est le Reali di Francia n'a qu'une faible ressemblance avec le fait historique, et seulement dans la mesure où les événements médiévaux mérovingiens peuvent être affirmés avec certitude. Ce que la chronique de la vie de Berthe fait, c'est révéler l'histoire d'une femme persécutée comme c'est le cas des légendes mariales. La longueur du conte de Berthe, en revanche, se distingue à la fois des légendes mariales contemporaines et des contes de fées ultérieurs, tout comme le fait sa parenté avec le panthéon chrétien pour sa résolution narrative. Son alignement avec les contes de fées ultérieurs vient du noyau de motifs propres au conte de fées qui ont survécu des centaines d'années et qui sont restés intacts, même en passant à travers toute une série de genres littéraires.

\section{Conclusion}

Dans cet article j'ai travaillé avec un échantillon d'histoires médiévales pour pénétrer dans le monde médiéval étranger du contage. Les contes de fées tels que nous les entendons aujourd'hui n'existaient pas encore, affirmation qui est tout à fait conforme avec les conclusions d'Albert Wesselski en I93I, de même que celles de Maren Clausen-Stolzenburg qui a écrit deux générations plus tard, en $1995^{49}$.

Les spécialistes de l'histoire du conte de fées attribuent depuis longtemps la transmission des contes, ou des éléments de contes depuis le monde antique, au Moyen Âge et aux périodes pré-moderne et moderne à la transmission orale non documentée. Rarement, sinon jamais, ont-ils considéré le rôle au Moyen Âge des prêcheurs comme liens entre le monde antique et le monde médiéval, ou comme sources de la connaissance populaire des motifs qui ont été réutilisés par la suite dans les contes de fées. Cependant, le lien vérifiable qui existe entre les motifs antiques de Johannes Gobi et les histoires médiévales explique ce phénomène avec plus de persuasion.

Ce que rapportent les recueils de contes exemplaires et leur usage par les prêcheurs itinérants, aussi bien que par les prêtres des églises, témoigne de l'existence d'une institution bien organisée et puissante qui a renforcé ce phénomène de transmission, à savoir celle de l'église et son rôle international au Moyen Âge. Les conséquences sur le contage ont été considé-

49. A. Wesselski, Versuch einer Theorie des Märchens (Reichenberg, Sudetendeutscher Verlag Franz Kraus [Prager Deutsche Studien, 45], 193I). Maren Clausen-Stolzenburg a abouti à la même conclusion dans Märchen und mittelalterliche Literaturtradition (Heidelberg, C. Winter, 1994). 
rables. Les contes dont on a discuté ici ont tous été écrits avant la moitié $\mathrm{du} \mathrm{Xv}^{\mathrm{e}}$ siècle. Cette date, quoique constituant une charnière littéraire non conventionnelle dans la chronologie de l'écriture au Moyen Âge, correspond à des changements littéraires significatifs dans le domaine des récits brefs. Au siècle qui a suivi, entre I450 et I550, il y a eu un profond changement dans la manière dont les femmes ont été décrites dans les récits brefs, sur le sujet des relations sexuelles en dehors du mariage. Les contes brefs qui parlent de la sexualité féminine à cette époque ont des issues profondément différentes de ceux qui ont été composés après I550, en ce qu'ils racontent une sorte différente d'histoires. La période I45O-I550 a aussi été témoin d'un changement soudain dans le genre d'histoires qui se racontaient, avec les premiers balbutiements dans l'histoire littéraire européenne d'un nouveau type d'intrigue, celui du conte d'ascension. Les deux courants ont ensuite fusionné quand les héroïnes ont été dénuées de leur puissance dans les récits brefs nouvellement écrits entre I450 et I550, et qu'on est entré dans le monde menaçant des contes de fées d'ascension qui a émergé en Italie avec les Piacevoli Notti (I55I, I553) de Giovan Francesco Straparola. 